\title{
The Institute of Circulatory and Respiratory Health Strategic Plan for 2013-2016 Executive Summary
}

\author{
Jean L Rouleau MD FRCPC ${ }^{1,2}$, Pierre Boyle PhD MHA², Ilana K Gombos PhD², Jennifer L Ralph BA ${ }^{2}$, \\ Lindsay D Jacobi Cadete MSc BSc${ }^{2}$, Maryse Desjardins ${ }^{1,2}$, Caroline Wong BA ${ }^{2}$
}

T he Institute of Circulatory and Respiratory Health (ICRH) - 1 of the 13 Institutes of the Canadian Institutes of Health Research (CIHR) - supports research that focuses on cardiovascular health, respiratory health, blood and blood vessels, stroke, critical/intensive care, and sleep/circadian rhythms. Together, the disease areas that are covered under the mandate of the ICRH represent conditions with the largest health, societal, and economic burden of any of the other Institutes within CIHR. This reality and the extraordinary diversity of these medical conditions present a major operational challenge for the ICRH. Despite these difficulties and limited resources, the Institute's 2013-2016 Strategic Plan1 demonstrates a commitment to ensuring that research excellence, capacity, competitiveness, innovation, and impact are enhanced across all research fields affiliated with the Institute. Moreover, building on previous successes, the ICRH continues to nurture past collaborations and work to establish new meaningful national and international alliances with the research community, partners, and other stakeholders to develop interdisciplinary, integrative health research that reflects Canada's emerging health needs, gaps, and opportunities. The ICRH also strongly supports partnerships with relevant stakeholders to accelerate the transfer of new knowledge into benefits for Canadians. Notably, patient engagement is an important priority that will serve as 1 of the major building blocks of all clinically related initiatives of the ICRH.

Based on identified gaps, opportunities, and feedback received from the International External Review Panel,2 ICRH research communities and partners, and in alignment with the CIHR 2014-2018 Strategic Plan, the ICRH identified 4 research priorities for 2013-2016.

The first priority focuses on enhancing capacity, competitiveness, and impact of our communities through networking. This objective is consistent with the importance of aligning with CIHR's largest comprehensive Roadmap Signature Initiative (RSI) the Strategy for Patient-Oriented Research (SPOR). In 2013, the Institute launched the ICRH Emerging Networks program in 3 gap areas, funding the Canadian Vascular Network, Canadian Respiratory Research Network, and the Canadian Stroke Prevention Intervention Network. In addition, through focused community development programs in critical care, stroke treatment and recovery, sleep and circadian rhythms, blood diseases, and resuscitation clinical research, the ICRH has targeted 5 additional research communities in need of networking. Aligned with CIHR's Patient Engagement Framework,3 the ICRH will use specific actions and tools to enhance patient engagement in health research, implement patient engagement as a core priority in strategic initiatives, and offer programs that will allow patients to be true partners in research. Finally, the ICRH is continuing its collaboration with the National Heart, Lung, and Blood Institute of the National Institutes of Health in the ongoing support of 2 large Canada-US networks-the Resuscitation Outcomes Consortium and the Cardiothoracic Surgical Network, and will explore other international network opportunities.
The second priority of the ICRH targets training, mentoring, and early career development to promote capacity-building-areas with an identified need. Recently, data have shown that the growth of funding within the ICRH's mandated research areas has been slower than in all other areas at the CIHR, in particular for the cardiovascular and respiratory communities (Fig. 1A). Evidence suggests that this is primarily due to declining numbers of young investigators (Fig. 1B). The development of research capacity related to health systems/services and population/public health has been identified as requiring particular attention. In the upcoming years, the ICRH will work closely with relevant CIHR Institutes and various working groups to address this important and persistent capacity problem, focusing on training/mentoring issues and issues affecting the career success of young investigators. In 2014, the ICRH facilitated the development of the Emerging Research Leaders Initiative, which was launched as a joint initiative between the Heart and Stroke Foundation and the Canadian Lung Association-2 of the ICRH's key partners. The ICRH will also continue its collaborative work with key stakeholders to develop a comprehensive training and mentoring strategy that will directly align with partner-led programs. A recent national ICRH survey (data not yet published) also confirmed the need for improved mentoring and the ICRH will work with its networks, partners, and the CIHR to improve the quality of mentoring based on current best practices.

The third priority of the ICRH focuses on the expansion of clinical research opportunities, health systems/services research, and population health through cohort linkage and data enhancement. This initiative will provide Canadian scientists with a unique opportunity to boost knowledge development and translation at a fraction of the cost of developing new cohorts and will enable Canada to develop better population-based research, and design individualized and community-based prevention and intervention programs. In addition, this initiative will contribute to improving the potential for health and health systems research by creating a pool of population-based data that can be linked to Canada's uniquely powerful administrative databases. Data enhancement and linkage will also directly assist in gathering and analyzing data related to Canada's Aboriginal people and other vulnerable populations that are presently lacking. Finally, by helping to address issues such as data storage and access, this initiative will support research on ethics, and increase the use and sustainability of existing cohorts. Along with the CIHR and its other Institutes, the ICRH is helping to coordinate disparate and multidisciplinary communities to bridge gaps and identify ways for harmonizing and sharing population-relevant data sets and their efficient utilization. In the context of the increasing prevalence of cardiovascular and respiratory risk factors, many of which are also risk factors for other serious chronic diseases, this initiative provides a unique opportunity for the ICRH community to link with other research communities in building tools to fuel clinical research and population health, health services, and policy research. Through the Environments and Health Signature Initiative, the ICRH is coleading the Data Platforms component,

\footnotetext{
${ }^{1}$ Montreal Heart Institute, Montreal, Quebec; ${ }^{2} \mathrm{CIHR}$ Institute of Circulatory and Respiratory Health, Ottawa, Ontario, Canada

Correspondence: Ms Caroline Wong, CIHR Institute of Circulatory and Respiratory Health, 5000 Belanger St - S2580, Montreal,

Quebec H1T 1C8, Canada. Telephone 514-593-7433, fax 514-376-7070, e-mail caroline.wong@icm-mhi.org

Received for publication October 4, 2014, Accepted October 9, 2014
} 


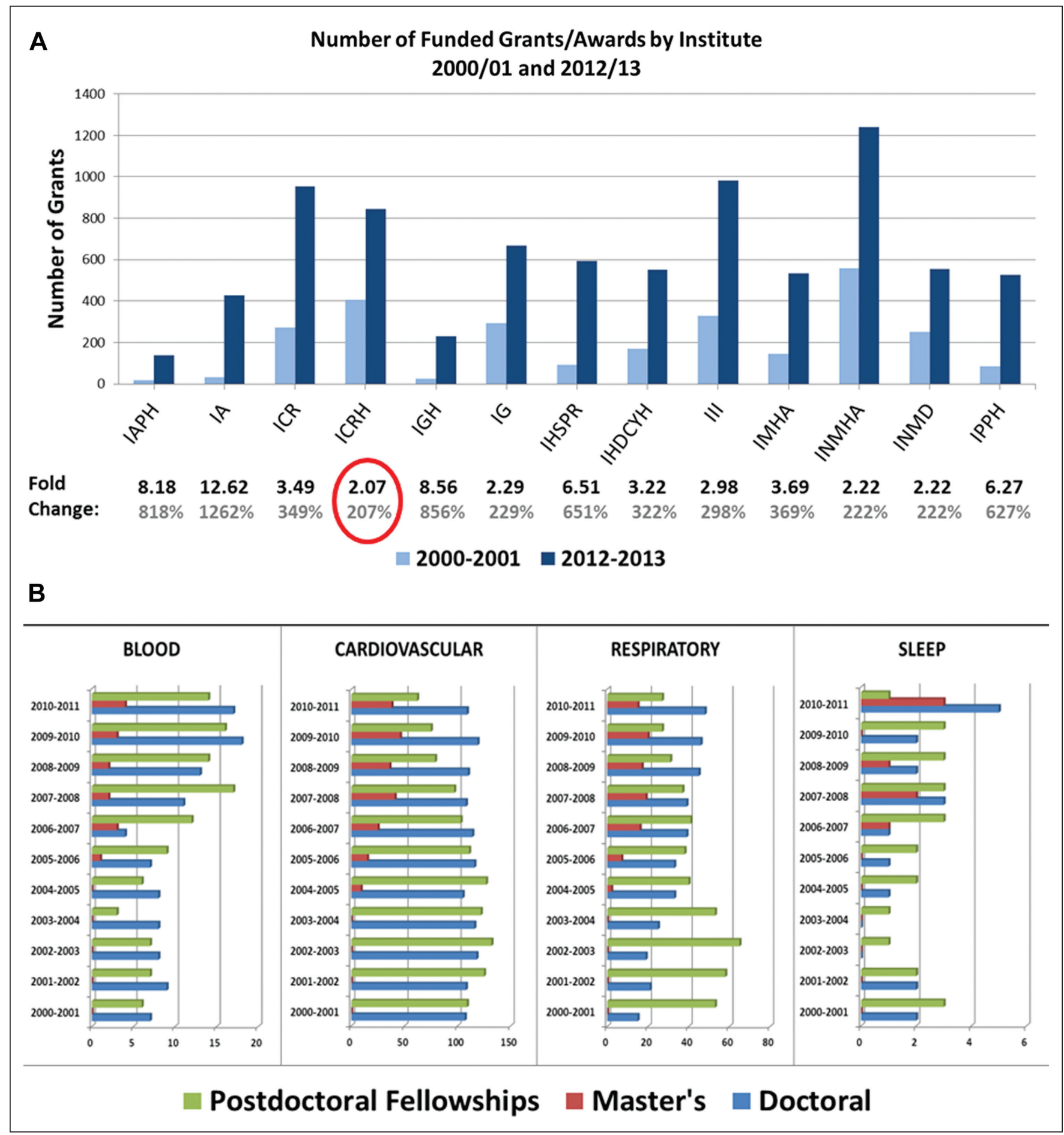

Figure 1) (A) Relative growth in number of funded grants/awards from 2000/2001 to 2012/2013 according to CIHR Institute. (B) Evolution in number of funded trainees, from 2000 to 2011, according to ICRH mandated field. CIHR, Canadian Institutes of Health Research; IA, Institute of Aging; IAPH, Institute of Aboriginal Peoples' Health; ICR, Institute of Cancer Research; ICRH, Institute of Circulatory and Respiratory Health; IG, Institute of Genetics; IGH, Institute of Gender and Health; IHDCYH, Institute of Human Development, Child and Youth Health; IHSPR, Institute of Health Services and Policy Research; III, Institute of Infection and Immunity; IMHA, Institute of Musculoskeletal Health and Arthritis; INMD, Institute of Nutrition, Metabolism and Diabetes; INMHA, Institute of Neurosciences, Mental Health and Addiction; IPPH, Institute of Population and Public Health. Modified and reproduced from Figures 3 (panel A) and 5 (panel B) in the CIHR Institute of Circulatory and Respiratory Health Strategic Plan for 2013-2016, with permission from the Canadian Institutes of Health Research, 2014.1

which will provide funding opportunities for enhancing Canadian health cohorts with environmental, microbiome, and other data.

The Institute identified contribution to the advancement of relevant CIHR RSIs as its fourth priority. These large-scale, multi-Institute programs bring the Canadian scientific community together and will transform research in selected areas on a national and international scale. Importantly, a number of these comprehensive programs, such as the Strategy for Patient-Oriented Research RSI, directly align with ICRH priorities; thus, the ICRH will work closely with the CIHR and its Institutes to advance these major initiatives. To support the 
Inflammation in Chronic Disease RSI, the ICRH is funding research on inflammation and respiratory diseases through the Health Challenges in Chronic Disease program. Moreover, the ICRH supports the Canadian National Transplant Research Program in partnership with the CIHR Institute of Infection and Immunity and multiple other partners. In support of the Personalized Medicine RSI, the ICRH contributed to specific projects that are aligned with the ICRH Emerging Networks and funded through the Genomics and Personalized Health program. In relation to the International Collaborative Research Strategy for Alzheimer's Disease RSI, the ICRH contributed funding toward the development of an integrated consortium-based research program that incorporates vascular elements and their role in the development of cognitive impairment. In collaboration with the CIHR Institute of Aboriginal People's Health and the Heart and Stroke Foundation, the ICRH has contributed to the development of a cohort aimed at thoroughly phenotyping a sample of Aboriginal individuals who live on reserves. This aligns with principals of the Pathways to Health Equity for Aboriginal Peoples RSI and will create an important research resource for developing and assessing programs for improving health in these communities. Finally, through the Environments and Health RSI and in alignment with its third priority, opportunities created by the ICRH will link a wide array of potential environmental health determinants to large, well-characterized cohorts that can link to health care utilization and outcomes, and be enriched with phenotypic, biomarker, and other information useful for assessing individual and population-level risk.

The ICRH is in a strong position to guide and shape the Canadian health research landscape. By working closely with its research communities and partners on Canada's research capacity through networking, training/mentoring/career development, and cohort linkage and data enhancement, the ICRH will continue to demonstrate its leadership and commitment to knowledge development and translation, and will be supporting research excellence aimed at reducing our burden of disease. Importantly, the ICRH will work closely with the CIHR to support and foster active engagement of patients at all levels of research within its communities to lead the way in transformative patient-oriented research.
TO READ THE FULL VERSION OF THE CIHR INSTITUTE OF CIRCULATORY AND RESPIRATORY HEALTH STRATEGIC PLAN FOR 2013-2016, PLEASE VISIT: http:// www.cihrirsc.gc.ca/e/documents/ICRH_Strategic_Plan_En_v8sept2014. pdf. A French version of this document is available as Supplementary material at www.pulsus.com. This document was revised and adapted, from text pages 3-5, Executive Summary, from the CIHR Institute of Circulatory and Respiratory Health Strategic Plan for 2013-2016, with permission from the Canadian Institutes of Health Research, 2014.1

FUNDING SOURCES: Funded by the Canadian Institutes of Health Research.

DISCLOSURES: The authors have no conflicts of interest to disclose.

SUPPLEMENTARY MATERIAL: To access the supplementary material accompanying this article, visit the online version of the Canadian Respiratory Journal at www.pulsus.com

\section{REFERENCES}

1. Institute of Circulatory and Respiratory Health. Institutes of the Canadian Institutes of Health Research. ICRH Strategic Plan for 2013-2016, September 8, 2014 Final Version. Available at: http:// www.cihr-irsc.gc.ca/e/documents/ICRH_Strategic_Plan_En_ v8sept2014.pdf. Accessed September 26, 2014.

2. Holgate S. 2011 International Review of the Canadian Institutes of Health Research. Expert Review Team Report for Institute of Circulatory and Respiratory Health. Available at: www.cihr-irsc. gc.ca/e/documents/ert-report_icrh-sholgate_e.pdf. Accessed September 26, 2014.

3. Canadian Institutes of Health Research. Strategy for PatientOriented Research. Patient Engagement Framework. Available at: http://www.cihr-irsc.gc.ca/e/documents/spor_framework-en.pdf. Accessed September 26, 2014.

This article is co-published in the Canadian Journal of Cardiology. Can J Cardiol 2014;30:1467-70. 


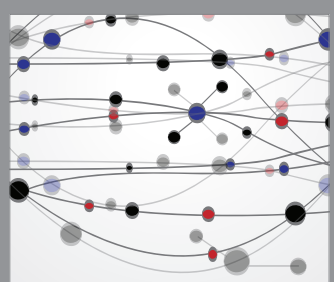

The Scientific World Journal
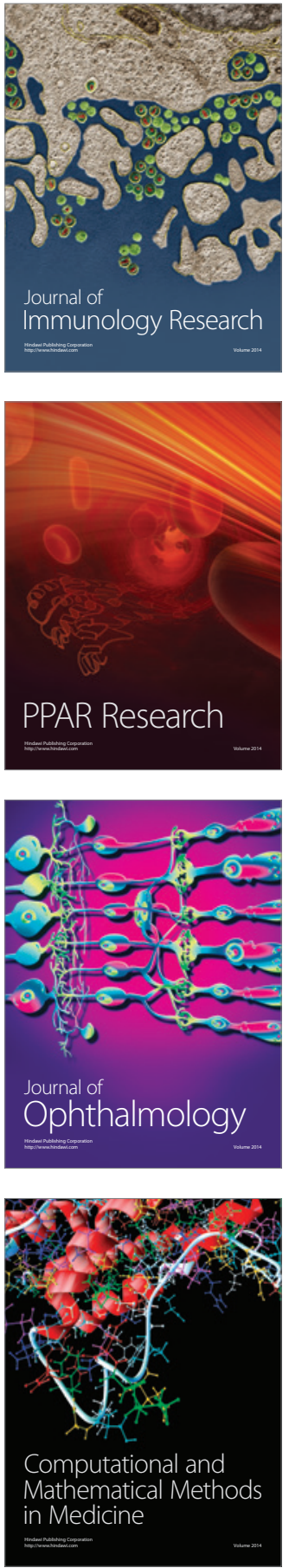

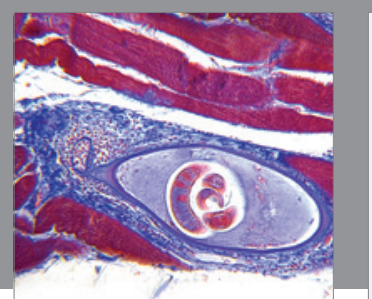

Gastroenterology Research and Practice

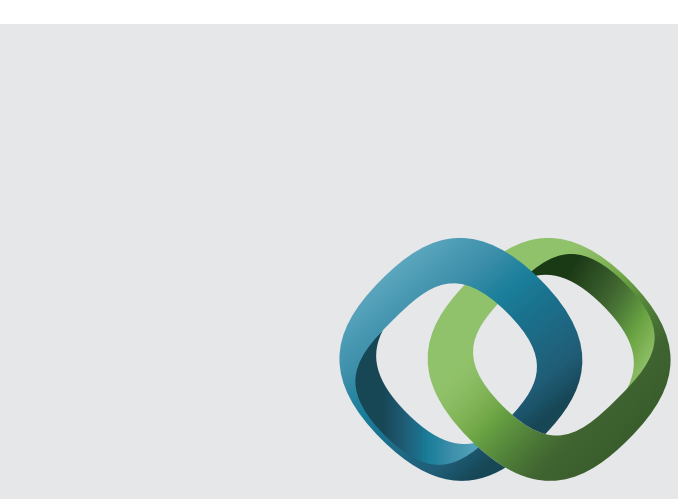

\section{Hindawi}

Submit your manuscripts at

http://www.hindawi.com
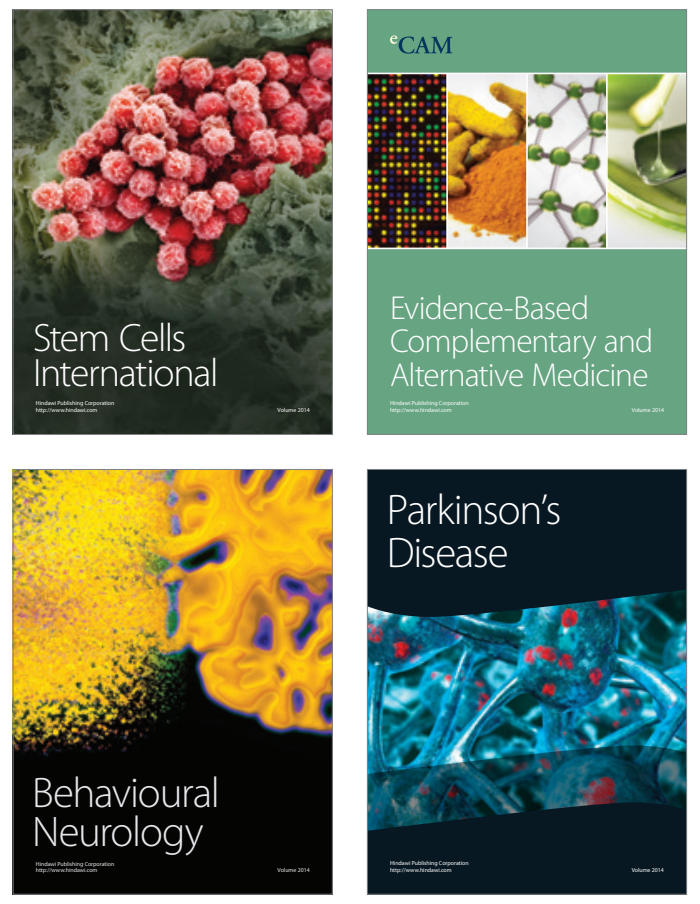
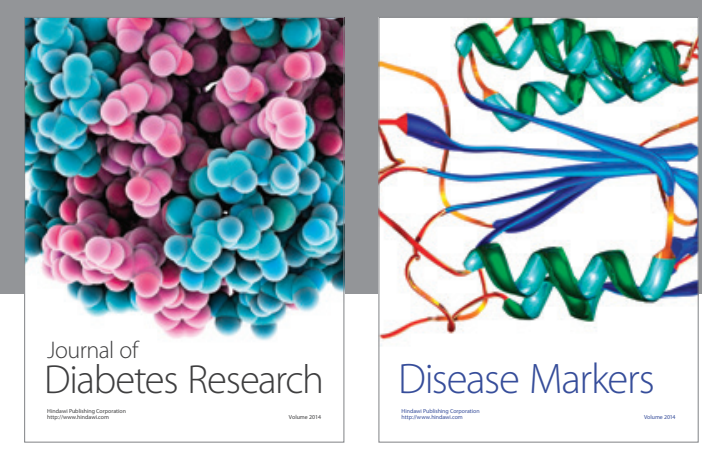

Disease Markers
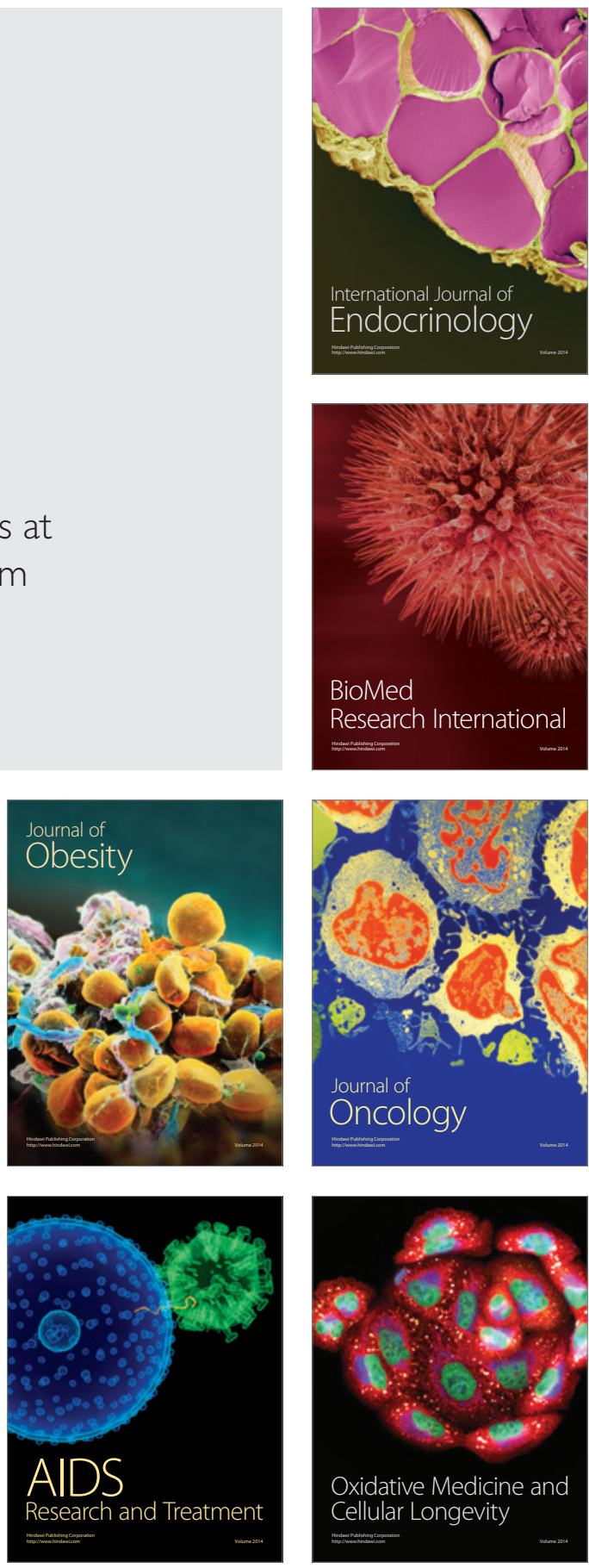